Gut, 1985, 26, 378-383

\title{
In vivo assessment of granulocyte migration to diseased bowel in Crohn's disease
}

\author{
S H SAVERYMUTTU, A M PETERS, J P LAVENDER, \\ V S CHADWICK, ${ }^{*}$ AND H J F HODGSON \\ From the Departments of Medicine and Diagnostic Radiology, Hammersmith Hospital, London
}

SUMMARY It has been suggested, on the basis of impaired granulocyte migration to skin windows, that there is a fundamental granulocyte defect in Crohn's disease. In vitro tests of granulocyte function have, however, failed to confirm this. We have studied granulocyte migration to inflamed bowel in Crohn's disease using a new approach which utilises dynamic gamma camera imaging after injection of ${ }^{111}$ In labelled autologous granulocytes. In 20 of 22 studies there was rapid migration to diseased bowel, compatible with no migration delay. Only two patients showed delays in migration of 12 and 15 minutes respectively, but neither had any clinical characteristics to distinguish them from the other 20 patients. This study shows that the majority of patients with Crohn's disease in relapse have rapid granulocyte migration to diseased bowel and provides evidence against a significant migration defect in this condition.

The similarity between the intestinal lesions in chronic granulomatous disease and Crohn's disease has led to the suggestion that defective granulocyte function may be involved in the pathogenesis of Crohn's disease. ${ }^{1}$ Support for this hypothesis is a striking impairment of in vivo granulocyte migration into inflammation induced in skin windows. ${ }^{2}$ Further studies have shown a delay of between two to four hours before leucocyte accumulation begins. $^{3}$ The possibility that identification of a granulocyte defect might provide the basis for specific therapy for Crohn's disease has led to a detailed assessment of granulocyte function in vitro. Because the results of such studies have been conflicting for both chemotaxis and phagocytosis, ${ }^{4}$ however, they have been of little value.

To determine whether there is a granulocyte migration defect in Crohn's disease there is a need for a direct in vivo assessment of granulocyte migration into diseased bowel. Recent work with ${ }^{111}$ indium labelled leucocytes has provided a possible approach. Thus, $\mathrm{we}^{5}$ and Segal et al, ${ }^{6}$ utilising a mixed ${ }^{111}$ indium labelled leucocyte preparation, have shown intense accumulation of

Address for correspondence: Dr S H Saverymuttu, Medicine II, St Georges Hospital Medical School, Cranmer Terrace, London SW17.

Received for publication 15 June 1984

* Present address: Wellcome Research Institute, University of Otago Medical School, Dunedin, New Zealand. labelled cells in Crohn's disease within two to four hours of injection. In a single patient in whom the kinetics of ${ }^{111}$ In labelled leucocytes were studied, a significant delay of 20-30 minutes before the start of granulocyte accumulation was shown in ileocaecal Crohn's disease. We have recently improved this technique by separating and labelling a pure granulocyte fraction without isolation from plasma. By using dynamic gamma camera imaging immediately after injection of labelled granulocytes we have monitored granulocyte migration into sites of inflammation. In the present study we have used this technique to measure the migration delay, if any, in Crohn's disease.

\section{Methods}

\section{PATIENTS}

Twenty two patients with clinically active Crohn's disease were studied. None had received any medication in the previous four months. The diagnosis was based on typical clinical and radiological features and, in 15 patients, confirmed by histology. In 12 patients, the disease involved the ileum alone in five, the ileum and colon, and five, the colon alone. Apart from ankylosing spondylitis in one patient, there were no extra intestinal complications. The Table summarises patient details. 
Table Clinical details and results in patients studied

\begin{tabular}{|c|c|c|c|c|c|c|}
\hline Patient & $\begin{array}{l}\text { Disease } \\
\text { distribu- } \\
\text { tion }\end{array}$ & $C D A I$ & $\begin{array}{l}E S R \\
(m m \text { fall } \\
\text { in 1st } h)\end{array}$ & $\begin{array}{l}B-b / A \\
\text { (\% per } \\
\text { min) }\end{array}$ & $A / a$ & $\begin{array}{l}D \\
(\text { min })\end{array}$ \\
\hline 1 & Ileal & 224 & 66 & $3 \cdot 8$ & $1 \cdot 15$ & 10 \\
\hline 2 & Ileal & 211 & 31 & $0 \cdot 9$ & 1.08 & 10 \\
\hline 3 & Ileocolonic & 190 & 38 & $1 \cdot 8$ & $1 \cdot 15$ & 10 \\
\hline 4 & Ileal & 156 & 16 & 0.9 & 1.04 & 10 \\
\hline 5 & Colonic & 348 & 100 & $2 \cdot 9$ & $1 \cdot 15$ & 10 \\
\hline 6 & Ileocolonic & 159 & 35 & $1 \cdot 2$ & $1 \cdot 12$ & 10 \\
\hline 7 & Colonic & 162 & 33 & $2 \cdot 8$ & 1.08 & 10 \\
\hline 8 & Ileal & 178 & 18 & $3 \cdot 9$ & 1.03 & 10 \\
\hline 9 & Ileal & 331 & 15 & $2 \cdot 6$ & 1.02 & 5 \\
\hline 10 & Ileal & 280 & 90 & $6 \cdot 4$ & $1 \cdot 13$ & 5 \\
\hline 11 & Ileal & 193 & 31 & $2 \cdot 6$ & $1 \cdot 12$ & 5 \\
\hline 12 & Ileal & 177 & 20 & $3 \cdot 9$ & $1 \cdot 37$ & 5 \\
\hline 13 & Ileal & 188 & 53 & $2 \cdot 8$ & 1.40 & 5 \\
\hline 14 & Colonic & 183 & 39 & $1 \cdot 6$ & $1 \cdot 30$ & 5 \\
\hline 15 & Colonic & 216 & 28 & $1 \cdot 6$ & $1 \cdot 1$ & 5 \\
\hline 16 & Ileal & 250 & 72 & $1 \cdot 0$ & $1 \cdot 2$ & 5 \\
\hline 17 & Ileal & 202 & 35 & $4 \cdot 0$ & $1 \cdot 14$ & 5 \\
\hline 18 & Ileal & 157 & 45 & $5 \cdot 6$ & $0 \cdot 83$ & 12 \\
\hline 19 & Colonic & 262 & 56 & $3 \cdot 0$ & 0.94 & 15 \\
\hline 20 & Colonic & 208 & 34 & $2 \cdot 4$ & $0 \cdot 83$ & 5 \\
\hline 21 & Colonic & 158 & 73 & $1 \cdot 0$ & $1 \cdot 10$ & 5 \\
\hline 22 & Colonic & 249 & 52 & 1.5 & $1 \cdot 24$ & 5 \\
\hline
\end{tabular}

B-b/A, A/a and D - see Fig. 1a. CDAI-Crohn's disease activity index.

LABELLING TECHNIQUES

Granulocytes were isolated in plasma enriched density gradient media and subsequently labelled in plasma with ${ }^{111}$ In tropolonate as previously described. $^{7}$

\section{IMAGING}

The labelled granulocytes were injected with the patient supine, positioned beneath a gamma camera (IGE 400T) interfaced to a computer (MDS $\mathrm{A}^{2}$ ). Dynamic imaging using a frame time of $15 \mathrm{sec}$ from 0-5 min and one minute subsequently was performed over a period of $40 \mathrm{~min}$ after injection. For the first 10 minutes (in the initial eight patients) or first five minutes (in the subsequent 14 patients) the camera was positioned over the chest, in order to record lung activity, and then moved to the abdomen to record activity from the site of diseased bowel. Time activity curves were constructed over the chest for the first five or $\mathbf{1 0}$ minutes and over the site of inflamed bowel and a control abdominal region for either the subsequent 35 or 30 minutes. In most cases the area of inflammation was not central, and the control region was taken symmetrically from the opposite side. In two cases with pancolitis (patients 21, 22) and one case (patient 20) with a diseased segment of transverse colon the control region was not symmetrical and selected from an uninvolved area of the abdomen with an attempt to ensure the inclusion of similar amounts of bone marrow and major blood vessels. The control and test regions had areas of equal size.

DATA ANALYSIS

Based on dynamic imaging over the lungs it was established in each case that pulmonary leucostasis was absent in that by five minutes the count rate over the lungs had fallen to less than $40 \%$ of initial maximal value and by $10 \mathrm{~min}$ to $30 \%$.

The counts collected in each minute over the test and control regions of the abdomen were subjected to computerised least squares linear regression analysis using the MINITAB programme. The significance of the difference between the slopes of the test and control regions was calculated using the respective standard deviations of the slopes. An estimate of the rate of accumulation of activity in the inflammatory site was made by subtracting the slopes of the control and test regions and expressing the difference in terms of the zero time intercept of the test region. The corrected slope $(\mathrm{B}-\mathrm{b}) / \mathrm{A}$, is the early granulocyte accumulation index (EGAI) and gives the rate of accumulation of granulocytes in inflamed bowel as a fraction of the zero time count per unit time (Fig. 1a). D, the maximum estimated migration delay, is the interval between injection and the time at which the count rate over the test region starts to rise. If there was no delay before granulocyte accumulation began the ratio of the extrapolated zero time intercepts of the test and control regions $(\mathrm{A} / \mathrm{a})$ would represent the relative blood pools of each region, and should be equal to or greater than unity. If there was a significant delay, the ratio of the extrapolated zero time intercepts would be expected to fall below unity (Fig. 1b).

\section{Results}

All 22 of the patients had positive scans within 40 minutes of injection of the labelled granulocytes (Fig. 2a). In several cases abnormal activity could be seen at $10 \mathrm{~min}$ (Fig. 2b). In 20 patients the count rate was rising over the test region at the start of the abdominal dynamic imaging (which in 12 cases was at five minutes and in the other eight cases at 10 min). An example of such a dynamic pattern is shown in Fig. 3. This study shows that the count rate was rising over the test region at five minutes and shows that this rise cannot be accounted for by background accumulation as the control region showed minimal change. In two patients (18 and 19) the count rate remained constant over the test region until 12 minutes and 15 minutes respectively after injection and then began to rise. An example 


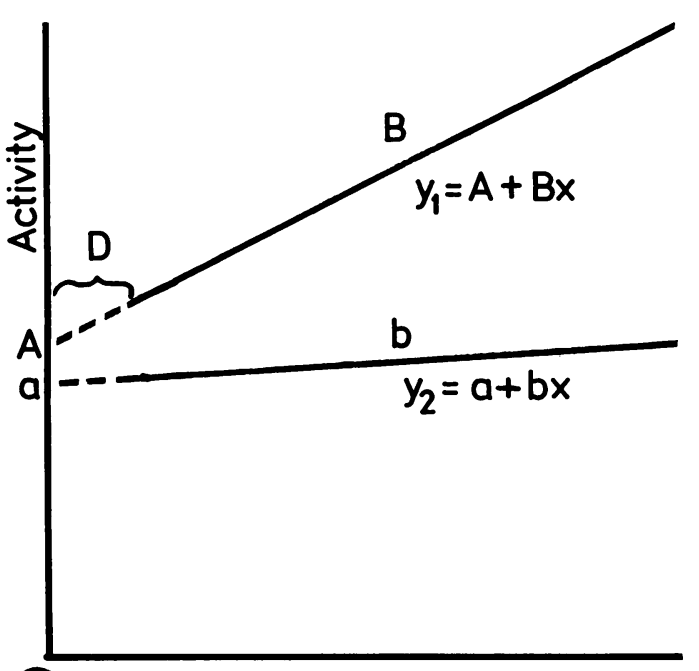

(a) Time from injection

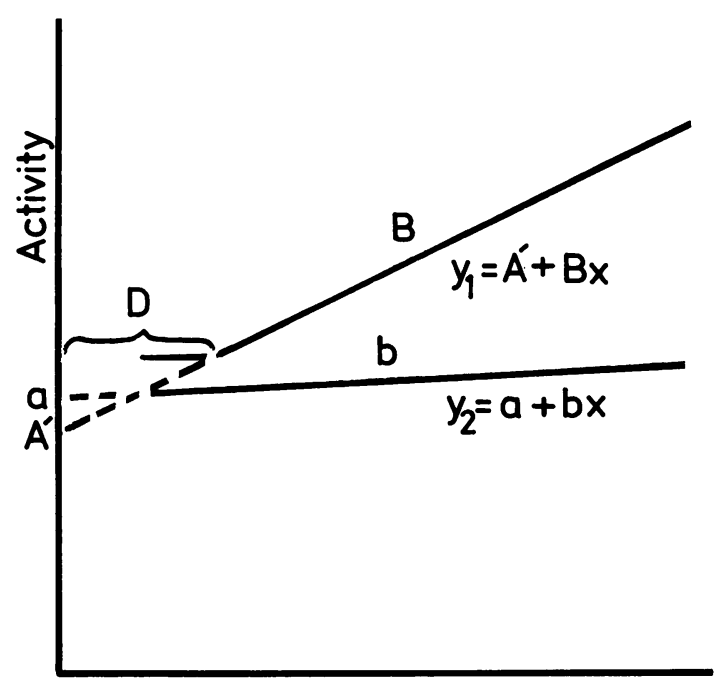

Time from injection

Fig. 1 Method of determining migration delay and corrected gradient of uptake of activity. (a) Maximum estimated migration delay $(D)$ less than five minutes. $y_{1}$ and $y_{2}$ are the count rates recorded over the test and control regions respectively. A and a are the corresponding zero time intercepts and $B$ and $b$ the gradients. The corrected slope is equal to $B-b / A$. A/a is greater than unity. (b) Maximum estimated migration delay (D) equals 10 minutes. $y_{1}, y_{2}, B, b$ and $a$ as in Fig. 1a. $A^{\prime}$ (zero time intercept of test slope) is less than a because of the migration delay of 10 minutes and so $A^{\prime} / a$ is less than unity.

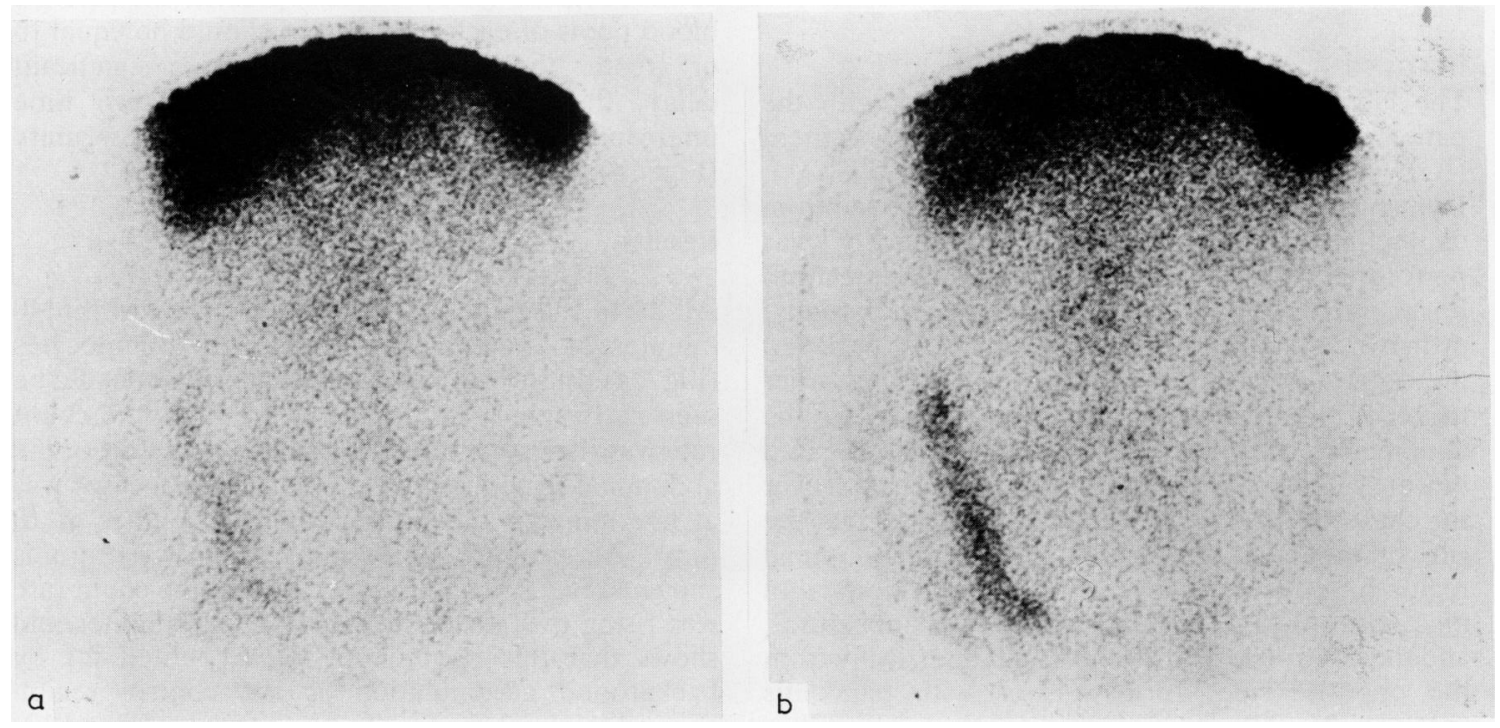

Fig. 2 (a) ${ }^{111}$ Indium granulocyte scan in patient 12 at 40 minutes showing abnormal activity in the right iliac fossa corresponding to ileal Crohn's disease. (b) Scan taken between 5 and 9 minutes already showing faint activity in the right iliac fossa. 


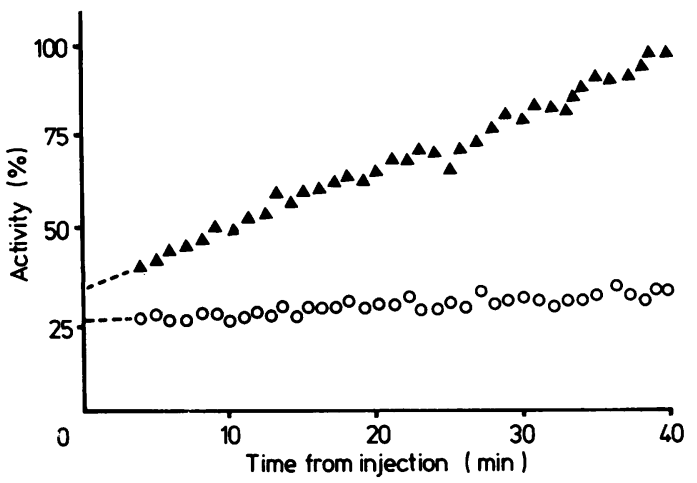

Fig. 3 Dynamic sequences showing a migration delay of less than five minutes in patient 12. Ordinate: counts collected in one minute frames. Abscissa: time from injection. $\boldsymbol{\Delta}$ = inflammatory focus; $\bigcirc=$ control region.

of this dynamic pattern is shown in Fig. 4. The Table summarises the values of the EGAI [(B-b)/A], A/a, and $D$, the maximum estimated granulocyte migration delay.

In the studies where localisation was in progress at the start of the dynamic imaging the maximum estimated delay in granulocyte migration was 10 minutes in patients $1-8$ and five minutes in patients 8-17. The ratios of the extrapolated zero time intercepts of $\mathrm{A}$ and a were greater than unity in all 17 patients in whom symmetrical test and control

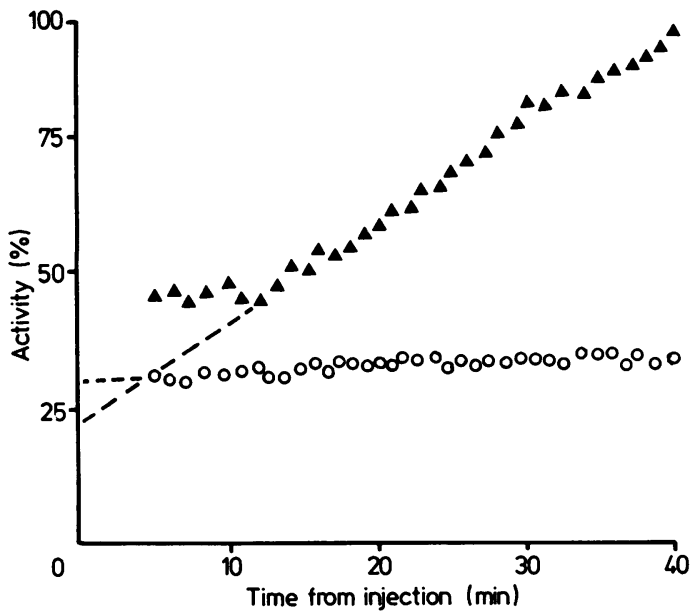

Fig. 4 Dynamic sequences showing a migration delay of 12 minutes in patient 18. Ordinate: counts collected in one minute frames. Abscissa: time from injection. $\mathbf{\Delta}=$ inflammatory focus; $\bigcirc=$ control region. regions were available (mean $1 \cdot 15 \pm$ SD $0 \cdot 11$ ). Only one patient $(20$, with the isolated segment of transverse colon involved, and an asymmetric control region, had an $\mathrm{A}^{\prime} / \mathrm{a}$ ratio of less than unity. In the two patients where granulocyte accumulation was not in progress at the start of dynamic imaging there were significant delays of 12 and 15 minutes. In both cases symmetrical control regions were analysed and the ratios of the extrapolated zero time intercepts were less than unity. There were no clinical features to distinguish these two patients from the other 20 with regard to activity, distribution, or duration of disease.

The EGAI varied from 0.9 to $6.4 \%$ per minute. In all cases there was a significant difference $(p<0.001)$ between the slopes of the test and control areas.

\section{Discussion}

Crohn's disease is a chronic inflammatory condition which is characterised by acute relapses interspersed with periods of remission. It may be that a defective acute inflammatory reaction resulting from granulocyte dysfunction is the cause of this condition. Despite considerable study, however, both in vivo and in vitro, the available evidence is inconclusive.

Using a novel approach to assess granulocyte function in vivo, the present investigation showed rapid migration of labelled granulocytes to inflamed bowel in Crohn's disease, compatible, in the majority of patients, with no migration delay at all. The significance of the delayed granulocyte accumulation showed in two of the patients is not certain. The ratios of the extrapolated zero time intercepts of less than unity suggest that this was a true migration delay specially as all but one of the remaining patients had ratios greater than unity. In previous studies, based on mixed leucocytes labelled in saline media, the immediate pulmonary granulocyte sequestration that was apparent may have been the cause of delayed localisation. In the present study, however, dynamic imaging showed rapid pulmonary granulocyte transit.

The observation of rapid granulocyte accumulation in inflamed bowel is difficult to reconcile with the results of skin window studies. Although it is an elegant method to examine granulocyte function in vivo the skin window technique has several important drawbacks. Firstly it is intrinsically insensitive in defining the magnitude of a migration delay, because as granulocyte accumulation in skin windows is counted at intervals of an hour, the delay is approximated to the nearest hour. Secondly, factors apart from granulocyte migration, namely granulocyte adhesion and tissue generation of chemotactic factors, influence the results. Finally it 
is not known whether it is valid to extrapolate from induced skin inflammation to naturally occurring inflammation in intestinal tissue.

In vitro studies are almost equally divided in support of the presence or absence of granulocyte dysfunction in Crohn's disease. Five groups have shown normal in vitro chemotaxis ${ }^{2}$ 8-10 $^{-10}$ while six have found abnormalities, either reduction of cellular chemotaxis or presence of serum inhibitors. ${ }^{41-15}$ These discrepant findings do not appear to result from differences in disease activity, duration of treatment or site of disease. They may, however, be related to problems associated with in vitro tests. Thus, many workers have subjected granulocytes to extensive in vitro manipulation during the separation procedures which result in cell damage. This cell damage is often too subtle to be detected by in vitro function tests yet may profoundly influence in vivo kinetics. ${ }^{16}$ A further drawback with in vitro chemotaxis assays - for example, the Boyden Chamber, is that, by using a leading front method, a non-representative subpopulation might be assessed. It appears likely that the existence of a granulocyte defect in Crohn's disease will not be resolved by in vitro testing because of problems of individual test modifications and lack of standardisation.

An in vivo scanning technique offers several advantages over the standard skin window technique and in vitro chemotaxis assays. Thus, the ability of ${ }^{111}$ indium granulocytes to monitor bowel inflammation renders the results directly relevant to Crohn's disease. As the skin window technique examines the total time for (a) the generation of chemotactic factors, (b) subsequent intravascular margination, (c) extravascular migration and, (d) accumulation in skin filters, an impairment at any of these stages could produce abnormal results. Because ${ }^{1}$ indium granulocyte scanning assesses spontaneous bowel inflammation, it is not influenced by delays in the generation of chemotactic factors. Furthermore, accumulation is monitored dynamically minute by minute. Thus ${ }^{111}$ indium granulocytes are more specific than the skin window technique for examining intravascular granulocyte margination and extravascular migration, the first two steps necessary in the natural defence mechanism dealing with foreign material.

Although ${ }^{111}$ indium granulocyte scanning offers clear advantages over existing techniques available to examine granulocyte migration, there are important theoretical and practical limitations. Dynamic scanning is also a 'leading front' measurement and so may have a problem in common with in vitro assays. The linearity of the accumulation in inflamed bowel during the $40 \mathrm{~min}$ imaging period suggests that it is assessing a representative granulocyte population. A major limitation of this technique is that only patients with active Crohn's disease can be investigated, as those with inactive disease will not accumulate granulocytes. Thus the technique could fail to detect a subtle granulocyte migration defect if it was present only in Crohn's disease in remission and which in theory could allow the initiation of a chain of events leading to a normal acute inflammatory response, ultimately assessed by 111 indium granulocyte scanning. There is no evidence, however, either from in vitro or skin window studies that the migration defect is confined to quiescent disease. The technique is not applicable to other conditions where defective granulocyte chemotaxis has been demonstrated - for example, Hodgkin's disease ${ }^{17}$ sarcoidosis $^{18}$ and alcoholic cirrhosis ${ }^{19}$ unless there is a fortuitous complicating abscess. It is, therefore, very difficult to establish a normal range of values.

The results from the study suggests that in most patients with Crohn's disease in relapse there is rapid migration of granulocytes to inflamed bowel and provides no evidence to support impaired granulocyte function in this condition.

\section{References}

1 Ward M. Pathogenesis of Crohn's disease. Lancet 1977; 2: 903-5.

2 Segal AW, Loewi G. Neutrophil dysfunction in Crohn's disease. Lancet 1976; 2: 219-1.

3 Wandal JE, Binder H. Leucocyte function in Crohn's disease. Gut 1982; 23: 173-80.

4 Kirk AP, Casaw J, Fordham et al. Polymorphonuclear leucocyte function in ulcerative colitis and Crohn's disease. Dig Dis Sci 1983; 28: 236-48.

5 Saverymuttu SH, Peters AM, Lavender JP, Hodgson HJF, Chadwick VS. ${ }^{111}$ Indium autologous leucocytes in inflammatory bowel disease. Gut 1983; 24: 293-9.

6 Segal AW, Ensell J, Munro JM, Sarner M. Indium-IIItagged leucocytes in the diagnosis of inflammatory bowel disease. Lancet 1981; 2: 230-2.

7 Saverymuttu SH, Peters AM, Reavy HJ, Lavender JP. Measurement of granulocyte migration and accumulation in inflammation in man. Clin Exp Immunol 1983; 52: 607-12.

8 O'Morain C, Segal A, Walker D. Levi AJ. Abnormalities of neutrophil function do not cause the migration defect in Crohn's disease. Gut 1981; 22: 817-20.

9 D'Amelio R, Rossi P, Le Moli S, Ricci R, Montano S, Fallone F. In vitro studies of cellular and humoral chemotaxis in Crohn's disease using the agarose gell technique. Gut 1981; 22: 566-70. 
10 Rhodes JM, Jewell DP. Motility of neutrophils and monocytes in Crohn's disease and ulcerative activity. Gut 1983; 24: 73-7.

11 Patrone F, Dallegri F, Sacchetti C. Defective neutrophil chemotaxis corrected by levamisole in Crohn's disease. Acta Hepatogastroenterol (Stuttg) 1978; 25: 322-3.

12 Gnarpe $\mathrm{H}$, Persson S, Belsheim J. Influence of metronidazole and tinidazole on leucocyte chemotaxis in Crohn's disease. Infection 1978; 6: suppl 1: 5107-9.

13 Belsheim J, Gnarpe H, Persson S. Granulocyte chemotaxis in Crohn's disease. Acta Chir Scand 1981; 147: 197-200.

14 Worsae N, Johansen K, Christensen KC. Impaired in vitro function of neutrophils in Crohn's disease. Scand J Gastroenterol 1982; 17: 91-6.

15 Rhodes JM, Potter BJ, Brown DJC, Jewell DP. Serum inhibitors of leucocyte chemotaxis in Crohn's disease and ulcerative colitis. Gastroenterology 1982; 82: 132734.

16 Saverymuttu SH, Peters AM, Reavy HJ, Danpure HJ, Osman S, Lavender JP. Lung transit of ${ }^{111}$ Indiumlabelled granulocytes - relationship to labelling techniques. Scand J Haematol 1983; 30: 151-60.

17 Ward PA, Berendberg JL. Defective regulation of inflammatory mediators in Hodgkin's disease: supernormal levels of chemotactic inactivator. $N$ Engl J Med 1974; 290: 76-80.

18 Gange RW, Black MM, Carrington P, McKerrow R. Defective neutrophil migration in sarcoidosis. Lancet 1977; 2: 379-81.

19 Maderazo EG, Ward PA, Qunityiliani R. Defective regulation of chemotaxis in cirrhosis. $J$ Lab Clin Med $1975 ; 85: 621$. 